DOI: $10.17516 / 1997-1397-2021-14-1-12-20$

УДК $519.1+517.44+512.54$

\title{
Integral Representation and the Computation of Multiple Combinatorial Sums from Hall's Commutator Theory
}

\author{
Georgy P. Egorychev* \\ Sergey G. Kolesnikov ${ }^{\dagger}$ \\ Vladimir M. Leontiev ${ }^{\ddagger}$ \\ Institute of Mathematics and Computer Science, \\ Siberian Federal University, \\ Svobodny 79, Krasnoyarsk, 660041 \\ Russian Federation
}

Received 10.09.2020, received in revised form 10.10.2020, accepted 20.11.2020

\begin{abstract}
In this paper we prove a series of combinatorial identities arising from computing the exponents of the commutators in P. Hall's collection formula. We also compute a sum in closed form that arises from using the collection formula in Chevalley groups for solving B. A. F. Wehrfritz problem on the regularity of their Sylow subgroups.
\end{abstract}

Keywords: integral representation, method of coefficients, P. Hall's collection formula.

Citation: G. P. Egorychev, S. G. Kolesnikov, V. M. Leontiev, Integral Representation and the Computation of Multiple Combinatorial Sums from Hall's Commutator Theory, J. Sib. Fed. Univ. Math. Phys., 2021, 14(1), 12-20. DOI: 10.17516/1997-1397-2021-14-1-12-20.

\section{Introduction and main results}

In the paper [1], P. Hall proved the collection formula for the expression $(x y)^{n}$ and showed that the exponents of the commutators in that formula are expressed as linear combinations of binomial coefficients with integral non-negative coefficients. In connection with different applications of the collection formula, the exponents of some commutators were found in a form of combinatorial sums in $[2,3,4,5]$. In the paper [6], a parametrization of the uncollected part of Hall's collection formula was proposed. The parametrization can be used to express the exponents of the commutators in the form of multiple combinatorial sums. For this reason, the problem of computing obtained sums in closed form and also modulo prime $n$ arises.

In this paper we prove a series of identities that arise in our research, using the method of coefficients $[7,8,9]$, which is directly connected with the theory of residues in multidimensional complex analysis. We also compute one combinatorial sum that regularly arises in computing commutators in Chevalley groups.

\footnotetext{
*gegorych@mail.ru

†sklsnkv@mail.ru

${ }^{\ddagger}$ v.m.leontiev@outlook.com

(c) Siberian Federal University. All rights reserved
} 
It was shown in [6] that the exponent of the commutator $\left[\left[y,{ }_{u} x\right],\left[y,{ }_{v} x\right]\right], 1 \leqslant v<u \leqslant n-1$, in the collection formula is equal to

$$
\sum_{m=1}^{n-1} \sum_{k=1}^{v} \sum_{i=v-k}^{n-m-k}\left(\begin{array}{c}
n-m-i-1 \\
k-1
\end{array}\right)\left(\begin{array}{c}
i \\
u-k+1
\end{array}\right)\left(\begin{array}{c}
i \\
v-k
\end{array}\right)+\sum_{m=1}^{n-2} \sum_{k=m+1}^{n-1}\left(\begin{array}{l}
m \\
v
\end{array}\right)\left(\begin{array}{l}
k \\
u
\end{array}\right)
$$

The first multiple sum was transformed in [10] as follows:

$$
\sum_{k=1}^{v} \sum_{i=v-k}^{n-k-1}\left(\begin{array}{c}
n-i-1 \\
k
\end{array}\right)\left(\begin{array}{c}
i \\
u-k+1
\end{array}\right)\left(\begin{array}{c}
i \\
v-k
\end{array}\right)
$$

Since the product $\left(\begin{array}{c}n-i-1 \\ k\end{array}\right)\left(\begin{array}{c}i \\ u-k+1\end{array}\right)\left(\begin{array}{c}i \\ v-k\end{array}\right)$ is equal to zero if $n-i-1<k$, or $i<u-k+1$, or $i<v-k$, we finally rewrite sum (1) in the following way:

$$
\sum_{k=1}^{v} \sum_{i=\max (0, u-k+1, v-k)}^{n-k-1}\left(\begin{array}{c}
n-i-1 \\
k
\end{array}\right)\left(\begin{array}{c}
i \\
u-k+1
\end{array}\right)\left(\begin{array}{c}
i \\
v-k
\end{array}\right)+\sum_{m=1}^{n-2} \sum_{k=m+1}^{n-1}\left(\begin{array}{l}
m \\
v
\end{array}\right)\left(\begin{array}{l}
k \\
u
\end{array}\right) .
$$

In [10], a combinatorial identity was proved that transforms the first multiple sum in (3) into linear combination of binomial coefficients of the form $\left(\begin{array}{c}n \\ w\end{array}\right)$ with integral non-negative coefficients. This representation is useful for computing the sum modulo prime $n$. In the following theorem, we give a new, simpler proof of the identity (4).

Theorem 1. Suppose

$$
\begin{aligned}
& \Omega_{1}=\left\{n, u, v \in \mathbb{N}, k \in \mathbb{N}_{0} \mid n \leqslant u+1\right\}, \\
& \Omega_{2}=\left\{n, u, v \in \mathbb{N}, k \in \mathbb{N}_{0} \mid v \leqslant u+1\right\}, \\
& \Omega_{3}=\left\{n, u, v \in \mathbb{N}, k \in \mathbb{N}_{0} \mid k \leqslant u+1\right\} .
\end{aligned}
$$

If the parameters $n, u, v, k$ belong to $\Omega=\Omega_{1} \cup \Omega_{2} \cup \Omega_{3}$, then the following combinatorial identity holds:

$$
\begin{aligned}
\sum_{i=\max (0, v-k, u-k+1)}^{n-k-1}\left(\begin{array}{c}
n-i-1 \\
k
\end{array}\right)\left(\begin{array}{c}
i \\
u-k+1
\end{array}\right)\left(\begin{array}{c}
i \\
v-k
\end{array}\right)= & \\
= & \sum_{i=\max (0, v-u-1)}^{\min (v-k, n-u-2)}\left(\begin{array}{c}
v-k \\
i
\end{array}\right)\left(\begin{array}{c}
n \\
i+u+2
\end{array}\right)\left(\begin{array}{c}
u-k+i+1 \\
v-k
\end{array}\right) .
\end{aligned}
$$

Denote by $S$ and $T$, respectively, the left-hand and right-hand sides of identity (4). Here, the combinatorial sums $S=S(z)$ and $T=T(z)$ are integer-valued functions of the integral parameters $z=(n, k, u, v)$. In addition to computing the sum $S$, we faced a difficult and, possibly, new problem in combinatorial practice: to find all the values of the parameters $n, k, u, v$ for which the identity $S(z)=T(z)$ holds. In other words, the problem is to find all the solutions of the equation $S(z)-T(z)=0$. Theorem 1 partially solves this problem, since sufficient conditions on the parameters $n, k, u, v$, are found for which the identity (4) holds. Let us note that Theorem 1 was proved thanks, in large part, to the fact that the terms of the combinatorial sums $S(z)$ and $T(z)$ are non-negative.

Further on, in connection with computing the exponent of the commutator $\left[\left[y,{ }_{i+1} x\right],\left[y,{ }_{i} x\right],{ }_{r} x\right]$ in another collection formula, the following identity was proved in 
[6]:

$$
\sum_{s_{r-1}=1}^{n} \sum_{s_{r-2}=1}^{s_{r-1}} \ldots \sum_{s_{1}=1}^{s_{2}} \sum_{s=1}^{s_{1}}\left(\begin{array}{c}
\left(\begin{array}{c}
s \\
i+1
\end{array}\right) \\
2
\end{array}\right)=\frac{1}{2}\left[\left(\begin{array}{c}
n \\
i+r+2
\end{array}\right)-\sum_{s=0}^{n-r-i-2}\left(\begin{array}{c}
n-i-s-2 \\
l
\end{array}\right)\left(\begin{array}{c}
i+s+1 \\
i+1
\end{array}\right)^{2}\right] .
$$

The following theorem shows that an $r$-multiple sum of a more general form can be reduced to a 2-multiple sum with the Stirling numbers of the first kind.

Theorem 2. Suppose $r, m, n, i \in \mathbb{N}_{0}, r \geqslant 1$ and $s_{1}(m, r)$ are the Stirling numbers of the first kind. Then we have the following $r$-multiple formula of summation:

$$
\sum_{s_{r-1}=1}^{n} \sum_{s_{r-2}=1}^{s_{r-1}} \ldots \sum_{s=1}^{s_{1}}\left(\begin{array}{c}
\left(\begin{array}{c}
s \\
i+1
\end{array}\right) \\
m
\end{array}\right)=\frac{1}{m !} \sum_{j=0}^{n} \sum_{s=0}^{n-i-1} s_{1}(m, j)\left(\begin{array}{c}
r+n-i-s-2 \\
r-1
\end{array}\right)\left(\begin{array}{c}
i+s+1 \\
s
\end{array}\right)^{j} .
$$

In conclusion, for the root system of type $A_{n}$, we compute the following sum:

$$
\sum_{i=1}^{n} \sum_{j=1}^{n} r_{j i} K_{-\alpha_{j},-\beta} K_{\alpha, \alpha_{i}}
$$

where $\alpha_{1}, \ldots, \alpha_{n}$ are all simple roots of the root system $\Phi, \alpha, \beta \in \Phi^{+},\left(r_{j i}\right)$ is the Cartan matrix. The numbers $K_{r, s}\left(r, s \in \Phi^{+}\right.$or $\left.r, s \in \Phi^{-}\right)$are defined by the following equality with the structure constants of the Lie algebra of type $\Phi$ :

$$
K_{r, s}=\sum_{\left(q_{1}, \ldots, q_{t}\right)} N_{s, q_{1}} N_{s+q_{1}, q_{2}} \ldots N_{s+q_{1}+\ldots+q_{t-1}, q_{t}},
$$

where the summation is taken over all combinations of not necessarily different simple roots $q_{1}, \ldots, q_{t}$ such that $r=s+q_{1}+\ldots+q_{t}$ and for any integer $i, 1 \leqslant i \leqslant t-1$, the sum $s+q_{1}+\ldots+q_{i}$ is a root. If such sequences do not exist, then we put $K_{r, s}=0$ by definition.

The sum (5) regularly arises in computing commutators in Chevalley groups. The following theorem holds.

Theorem 3. Suppose the natural numbers: $p, q, k, m, n$ satisfy the inequalities: $1 \leqslant p<q \leqslant n+1$, $1 \leqslant k<m \leqslant n+1$. Then we have

$$
\sum_{i=1}^{n} \sum_{j=1}^{n}(-1)^{i+j} r_{i j}\left(\begin{array}{l}
m-k-1 \\
m-j-1
\end{array}\right)\left(\begin{array}{c}
q-p-1 \\
q-i-1
\end{array}\right)=\left(\begin{array}{c}
q+m-p-k \\
m-p
\end{array}\right),
$$

where $\left(r_{i j}\right)$ is the following square $n \times n$ matrix:

$$
\left(r_{i j}\right)=\left(\begin{array}{rrrrr}
2 & -1 & \ldots & 0 & 0 \\
-1 & 2 & \ldots & 0 & 0 \\
\vdots & \vdots & \ldots & \vdots & \vdots \\
0 & 0 & \ldots & 2 & -1 \\
0 & 0 & \ldots & -1 & 2
\end{array}\right) .
$$

\section{Proof of Theorem 1}

To prove Theorem 1 we need several lemmas. Let

$$
\Phi(w)=\Phi\left(w_{1}, w_{2}, w_{3}, w_{4}\right)=\sum_{n, u, v \in \mathbb{N}_{0}, k \in \mathbb{N}} S(n, u, v, k) w_{1}^{n} w_{2}^{u} w_{3}^{v} w_{4}^{k}
$$

be the generating function for the multiple sequence $S$. 
Lemma 1. The function $\Phi(w), w \in \mathbb{C}^{4}$, is rational and holomorphic in a neighborhood of zero and has the following form:

$$
\Phi(w)=\frac{w_{1}^{2} w_{3} w_{4}}{\left(1-w_{1}\right)\left(1-w_{1}\left(1+w_{2}\right)\left(1+w_{3}\right)\right)\left(1-w_{1}-w_{1} w_{2} w_{3} w_{4}\right)},
$$

where

$$
\left|w_{1} w_{2} w_{3} w_{4} /\left(1-w_{1}\right)\right|<1, \quad\left|w_{1}\left(1+w_{2}\right)\left(1+w_{3}\right)\right|<1
$$

Proof. We have

$$
\begin{aligned}
\Phi(w) & =\sum_{n, u, v \in \mathbb{N}_{0}, k \in \mathbb{N}} S(n, u, v, k) w_{1}^{n} w_{2}^{u} w_{3}^{v} w_{4}^{k}= \\
& =\sum_{n, u, v \in \mathbb{N}_{0}, k \in \mathbb{N}} w_{1}^{n} w_{2}^{u} w_{3}^{v} w_{4}^{k}\left(\sum_{i=\max (0, v-k, u-k+1)}^{n-k-1}\left(\begin{array}{c}
n-i-1 \\
k
\end{array}\right)\left(\begin{array}{c}
i \\
u-k+1
\end{array}\right)\left(\begin{array}{c}
i \\
v-k
\end{array}\right)=\right. \\
& =\sum_{n, u, v \in \mathbb{N}_{0}, k \in \mathbb{N}} w_{1}^{n} w_{2}^{u} w_{3}^{v} w_{4}^{k}\left(\sum_{i=0}^{n-k-1} \operatorname{res}_{x}\left\{\frac{(1-x)^{-k-1}}{x^{n-i-k}}\right\} \cdot \underset{y}{\operatorname{res}}\left\{\frac{(1+y)^{i}}{y^{u-k+2}}\right\} \cdot \underset{z}{\operatorname{res}}\left\{\frac{(1+z)^{i}}{z^{v-k+1}}\right\}\right)=
\end{aligned}
$$

we extend the summation over $i$ to infinity, adding zero terms, $|x|<1$,

$$
=\sum_{n, u, v \in \mathbb{N}_{0}, k \in \mathbb{N}} w_{1}^{n} w_{2}^{u} w_{3}^{v} w_{4}^{k} \underset{x, y, z}{\operatorname{res}}\left\{\frac{(1-x)^{-k-1}}{x^{n-k} y^{u-k+2} z^{v-k+1}} \cdot\left[\sum_{i=0}^{\infty}(x(1+y)(1+z))^{i}\right]\right\}=
$$

summing over $i,|x(1+y)(1+z)|<1$,

$$
\begin{aligned}
& =\sum_{n, u, v \in \mathbb{N}_{0}, k \in \mathbb{N}} w_{1}^{n} w_{2}^{u} w_{3}^{v} w_{4}^{k} \underset{x, y, z}{\operatorname{res}}\left\{\frac{(1-x)^{-k-1}}{x^{n-k} y^{u-k+2} z^{v-k+1}(1-x(1+y)(1+z))}\right\}= \\
& =\sum_{n, u, v \in \mathbb{N}_{0}} w_{1}^{n} w_{2}^{u} w_{3}^{v} \underset{x, y, z}{\operatorname{res}}\left\{\frac{(1-x)^{-2} w_{4}}{x^{n-1} y^{u+1} z^{v}(1-x(1+y)(1+z))} \sum_{k=1}^{\infty}\left(\frac{x y z w_{4}}{1-x}\right)^{k-1}\right\}=
\end{aligned}
$$

summing over $k,\left|x y z w_{4} /(1-x)\right|<1$,

$$
=\sum_{n, u, v \in \mathbb{N}_{0}} w_{1}^{n} w_{2}^{u} w_{3}^{v} \underset{x, y, z}{\operatorname{res}}\left\{\frac{(1-x)^{-1} w_{4}}{x^{n-1} y^{u+1} z^{v}(1-x(1+y)(1+z))\left(1-x-x y z w_{4}\right)}\right\}=
$$

summing over $n, u, v$ by the substitution rule [7, p. 13] and the change: $x=w_{1}, y=w_{2}, z=w_{3}$,

$$
=\frac{w_{1}^{2} w_{3} w_{4}}{\left(1-w_{1}\right)\left(1-w_{1}\left(1+w_{2}\right)\left(1+w_{3}\right)\right)\left(1-w_{1}-w_{1} w_{2} w_{3} w_{4}\right)} .
$$

The lemma is proved.

Let

$$
\Psi(w)=\Psi\left(w_{1}, w_{2}, w_{3}, w_{4}\right)=\sum_{n, u, v \in \mathbb{N}_{0}, k \in \mathbb{N}} T(n, u, v, k) w_{1}^{n} w_{2}^{u} w_{3}^{v} w_{4}^{k},
$$

be the generating function for the multiple sequence $T$.

Lemma 2. The function $\Psi(w), w \in \mathbb{C}^{4}$, is rational and holomorphic in a neighbourhood of zero and has the following form:

$$
\Psi(w)=\frac{w_{1}^{2} w_{3} w_{4}\left(1+w_{3}\right)\left(1-w_{1}\right)^{-1}\left(1-w_{1}\left(1+w_{2}\right)\left(1+w_{3}\right)\right)^{-1}}{\left(1-w_{1}+w_{3}-w_{1} w_{3}-w_{3} w_{4}-w_{1} w_{3} w_{4}-w_{2} w_{3}^{2} w_{4}\right.},
$$

where

$$
\left|\frac{w_{1} w_{3}\left(1+w_{3}\right)}{1-w_{1}-w_{1} w_{3}}\right|<1, \quad\left|\frac{w_{3} w_{4}\left(1-w_{1}-w_{1} w_{3}\right)}{\left(1-w_{1}\right)\left(1+w_{3}\right)}\right|<1 .
$$


Proof. We have

$$
\begin{aligned}
T & =\sum_{i=0}^{v-k}\left(\begin{array}{c}
v-k \\
i
\end{array}\right)\left(\begin{array}{c}
n \\
i+u+2
\end{array}\right)\left(\begin{array}{c}
u-k+i+1 \\
v-k
\end{array}\right)= \\
& =\sum_{i=0}^{v-k} \operatorname{res}_{x}\left\{\frac{(1+x)^{v-k}}{x^{i+1}}\right\} \cdot \operatorname{res}_{y}\left\{\frac{(1-y)^{-(i+u+2)-1}}{y^{n-(i+u+2)+1}}\right\} \cdot \underset{z}{\operatorname{res}}\left\{\frac{(1+z)^{u-k+i+1}}{x^{v-k+1}}\right\}= \\
& =\operatorname{res}_{x, y, z}\left\{\frac{(1-y)^{-u-3}(1+z)^{u-k+1}}{y^{n-u+3} z^{v-k+1}}\left[\sum_{i=0}^{v-k} \frac{(1+x)^{v-k}}{x^{i+1}}\left(\frac{y(1+z)}{1-y}\right)^{i}\right]\right\}=
\end{aligned}
$$

summing over $i$ by the substitution rule and the change: $x=y(1+z)(1-y)^{-1}$,

$$
\begin{aligned}
& =\operatorname{res}_{y, z}\left\{\frac{\left(1+y(1+z)(1-y)^{-1}\right)^{v-k}(1-y)^{-u-3}(1+z)^{u-k+1}}{y^{n-u-1} z^{v-k+1}}\right\}= \\
& =\operatorname{res}_{y, z}\left\{\frac{(1+y z)^{v-k}(1-y)^{-(v-k+u+3}(1+z)^{u-k+1}}{y^{n-u-1} z^{v-k+1}}\right\} .
\end{aligned}
$$

Therefore,

$$
\begin{aligned}
\Psi(w) & =\sum_{n, u, v \in \mathbb{N}_{0}, k \in \mathbb{N}} T(n, u, v, k) w_{1}^{n} w_{2}^{u} w_{3}^{v} w_{4}^{k}= \\
& =\sum_{n, u, v \in \mathbb{N}_{0}, k \in \mathbb{N}} w_{1}^{n} w_{2}^{u} w_{3}^{v} w_{4}^{k} \operatorname{res}_{y, z}\left\{\frac{(1+y z)^{v-k}(1-y)^{-(v-k+u+3} y^{u+2}(1+z)^{u-k+1}}{y^{n+1} z^{v-k+1}}\right\}=
\end{aligned}
$$

summing over $n$ by the substitution rule and the change: $y=w_{1}$,

$$
=\sum_{u, v \in \mathbb{N}_{0}, k \in \mathbb{N}} w_{1}^{u+2} w_{2}^{u} w_{4}^{k} \operatorname{res}_{z}\left\{\frac{\left(1+w_{1} z\right)^{v-k}\left(1-w_{1}\right)^{-(v-k+u+3}(1+z)^{u-k+1}}{z^{v-k+1}}\right\}=
$$

the change $t=z\left(1-w_{1}\right) / w_{3}\left(1+w_{1} z\right),|z| \ll 1$,

$$
=\sum_{u, v \in \mathbb{N}_{0}, k \in \mathbb{N}} w_{1}^{u+2} w_{2}^{u} w_{3}^{k} w_{4}^{k} \underset{z}{\operatorname{res}}\left\{\frac{\left(1-w_{1}\right)^{-k-2}\left(1+t w_{3}\right)^{u-k+1} t^{k+1}}{t^{v+1}\left(1-w_{1}-y w_{1} w_{3}\right)^{u-k+2}}\right\}=
$$

summing over $v$ by the substitution rule and the change: $t=1$,

$$
\begin{aligned}
& =\sum_{u \in \mathbb{N}_{0}, k \in \mathbb{N}} w_{1}^{u+2} w_{2}^{u} w_{3}^{k} w_{4}^{k} \frac{\left(1-w_{1}\right)^{-k-2}\left(1+w_{3}\right)^{u-k+1}}{\left(1-w_{1}-y w_{1} w_{3}\right)^{u-k+2}}= \\
& =\frac{w_{1}^{2}\left(1+w_{3}\right)}{\left(1-w_{1}\right)^{2}\left(1-w_{1}-w_{1} w_{3}\right)^{2}} \cdot \sum_{u=0}^{\infty}\left(\frac{w_{1} w_{2}\left(1+w_{3}\right)}{1-w_{1}-w_{1} w_{3}}\right)^{u} \sum_{k=1}^{\infty}\left(\frac{w_{3} w_{4}\left(1-w_{1}-w_{1} w_{3}\right)}{\left(1-w_{1}\right)\left(1+w_{3}\right)}\right)^{k} .
\end{aligned}
$$

Since (10) holds, it remains to use the summation formula for a geometric series to simplify the sums over $u$ and $k$. The lemma is proved.

Let us denote by $\Theta(w)$ the difference $\Phi(w)-\Psi(w)$. Then $\Theta(w)$ is not identically zero, is rational and holomorphic in a neighbourhood of zero, and has the following form:

$$
\Theta(w)=\frac{-w_{1}^{2} w_{3}^{2} w_{4}^{2}\left(1-w_{1}-w_{1} w_{2} w_{3} w_{4}\right)^{-1}}{\left(1-w_{1}\right)\left(1+w_{3}\right)-w_{3} w_{4}\left(1-w_{1}-w_{1} w_{3}\right)}
$$

where

$$
\left|\frac{w_{3} w_{4}\left(1-w_{1}-w_{1} w_{3}\right)}{\left(1-w_{1}\right)\left(1+w_{3}\right)}\right|<1 \quad\left|\frac{w_{1} w_{2} w_{3} w_{4}}{1-w_{1}}\right|<1, \quad\left|w_{i}\right| \ll 1, i=1,2,3
$$


Suppose

$$
\Theta(w)=\sum_{n, u, v \in \mathbb{N}_{0}, k \in \mathbb{N}} \theta(n, u, v, k) w_{1}^{n} w_{2}^{u} w_{3}^{v} w_{4}^{k}
$$

and

$$
\theta(n, u, v, k)=\operatorname{res}_{w_{1}, w_{2}, w_{3}, w_{4}}\left\{\frac{\Theta(w)}{w_{1}^{n+1} w_{2}^{u+1} w_{3}^{v+1} w_{4}^{k+1}}\right\}, \quad \forall n, u, v \in \mathbb{N}_{0}, \forall k \in \mathbb{N} .
$$

To conclude the proof of Theorem 1 it remains to show that if $n, u, v, k \in \Omega$, then $\theta(n, u, v, k)=0$ and, therefore, $S(n, u, v, k)=T(n, u, v, k)$.

We have

$$
\theta(n, u, v, k)=\operatorname{res}_{w}\left\{\frac{w_{1}^{2} w_{3}^{2} w_{4}^{2}\left(1-w_{1}\right)^{-1}\left(1-w_{1}-w_{1} w_{2} w_{3} w_{4}\right)^{-1}}{\left.w_{1}^{n+1} w_{2}^{u+1} w_{3}^{v+1} w_{4}^{k+1}\left(\left(1-w_{1}\right)\left(1+w_{3}\right)-w_{3} w_{4}\left(1-w_{1}-w_{1} w_{3}\right)\right)\right)}\right\} .
$$

By expanding the functions

$$
\left(1-w_{1}-w_{1} w_{2} w_{3} w_{4}\right)^{-1}, \quad\left(\left(1-w_{1}\right)\left(1+w_{3}\right)-w_{3} w_{4}\left(1-w_{1}-w_{1} w_{3}\right)\right)^{-1}
$$

in powers of $\left(w_{1} w_{2} w_{3} w_{4}\right)\left(1-w_{1}\right)^{-1}, w_{3} w_{4} f\left(w_{1}, w_{3}\right)$, respectively, where

$$
f\left(w_{1}, w_{3}\right)=\frac{\left(1-w_{1}-w_{1} w_{3}\right)}{\left(1-w_{1}\right)\left(1+w_{3}\right)},
$$

we obtain

$$
\theta(n, u, v, k)=\operatorname{res}_{w_{1}, w_{2} w_{3}, w_{4}}\left\{\frac{\left(1-w_{1}\right)^{-3}\left(1+w_{3}\right)^{-1}}{w_{1}^{n+1} w_{3}^{v+1} w_{4}^{k+1}} \cdot \sum_{j=0}^{\infty} w_{3}^{j} w_{4}^{j} f\left(w_{1}, w_{3}\right)^{j} \cdot \sum_{i=0}^{\infty}\left(\frac{w_{1} w_{2} w_{3} w_{4}}{1-w_{1}}\right)^{i}\right\} .
$$

Transforming the last expression into the form

$$
\underset{w_{1}, w_{3}, w_{4}}{\operatorname{res}}\left\{\frac{\left(1-w_{1}\right)^{-3}\left(1+w_{3}\right)^{-1}}{w_{1}^{n+1} w_{3}^{v+1} w_{4}^{k+1}} \cdot \sum_{j=0}^{\infty} w_{3}^{j} w_{4}^{j} f\left(w_{1}, w_{3}\right)^{j} \cdot \underset{w_{2}}{\operatorname{res}}\left\{\frac{1}{w_{2}^{u+1}} \sum_{i=0}^{\infty}\left(\frac{w_{1} w_{2} w_{3} w_{4}}{1-w_{1}}\right)^{i}\right\}\right\}
$$

and computing the residue at the point $w_{2}=0$, we get

$$
\theta(n, u, v, k)=\operatorname{res}_{w_{1}, w_{3}, w_{4}}\left\{\frac{\left(1-w_{1}\right)^{u-3}}{w_{1}^{(n-u-2)+1}} \cdot \frac{1}{\left(1+w_{3}\right)} \cdot \sum_{j=0}^{\infty} \frac{f\left(w_{1}, w_{3}\right)^{j}}{w_{3}^{(v-2-j-u)+1} w_{4}^{(k-u-j-2)+1}}\right\} .
$$

The residue at the point $w_{1}=0$ is equal to zero if $n-u-2<0$, i.e. $n \leqslant u+1$ (the case $\left.(n, u, v, k) \in \Omega_{1}\right)$. The residue at the point $w_{3}=0$ is equal to zero for every $j \geqslant 0$ if $v-2-j-u<0$, i.e. for $v \leqslant u+1$ (the case $\left.(n, u, v, k) \in \Omega_{2}\right)$. Finally, rewriting (14) as follows:

$$
\theta(n, u, v, k)=\operatorname{res}_{w_{1}, w_{3}}\left\{\frac{\left(1-w_{1}\right)^{u-3}}{w_{1}^{(n-u-2)+1}} \cdot \frac{1}{1+w_{3}} \cdot\left[\sum_{j=0}^{\infty} \frac{f\left(w_{1}, w_{3}\right)^{j}}{w_{3}^{(v-2-j-u)+1}} \cdot \underset{w_{4}}{\operatorname{res}}\left\{\frac{1}{w_{4}^{(k-u-j-2)+1}}\right\}\right]\right\},
$$

we see that the residue at the point $w_{4}=0$ is equal to zero for every $j \geqslant 0$ if $k<u+1$ (the case $\left.(n, u, v, k) \in \Omega_{3}\right)$. 


\section{Proof of Theorem 2.}

Let us recall the definition of the Stirling numbers of the first kind $s_{1}(m, r)([7]$, p. 272):

$$
a(a-1) \times \ldots \times(a-n+1)=\sum_{k=0}^{n} s_{1}(n, k) a^{k}, \quad \forall a \in \mathbb{R} .
$$

Thus, we have

$$
\begin{gathered}
\left(\begin{array}{c}
a \\
m
\end{array}\right)=\frac{a(a-1) \ldots(a-m+1)}{m !}=\frac{1}{m !} \sum_{j-0}^{m} s_{1}(m, j) a^{j}, \\
\left(\begin{array}{c}
\left(\begin{array}{c}
s \\
i+1
\end{array}\right) \\
m
\end{array}\right)=\frac{1}{m !} \sum_{j-0}^{m} s_{1}(m, j)\left(\begin{array}{c}
s \\
i+1
\end{array}\right)^{j} .
\end{gathered}
$$

Taking into account (16), we obtain

$$
\sum_{s_{r-1}=1}^{n} \sum_{s_{r-2}=1}^{s_{r-1}} \ldots \sum_{s=1}^{s_{1}} \frac{1}{m !} \sum_{j=0}^{m} s_{1}(m, j)\left(\begin{array}{c}
s \\
i+1
\end{array}\right)^{j}=\frac{1}{m !} \sum_{j=0}^{m} s_{1}(m, j)\left\{\sum_{s_{r-1}=1}^{n} \sum_{s_{r-2}=1}^{s_{r-1}} \ldots \sum_{s=1}^{s_{1}}\left(\begin{array}{c}
s \\
i+1
\end{array}\right)^{j}\right\} .
$$

Using the identity

$$
\sum_{s_{r-1}=1}^{n} \sum_{s_{r-2}=1}^{s_{r-1}} \ldots \sum_{s_{1}=1}^{s_{2}} \sum_{s=1}^{s_{1}}\left(\begin{array}{c}
s \\
i+1
\end{array}\right)^{m}=\sum_{s=0}^{n-i-1}\left(\begin{array}{c}
r+n-i-s-2 \\
r-1
\end{array}\right)\left(\begin{array}{c}
i+s+1 \\
s
\end{array}\right)^{m}, \quad m>1
$$

proved in [6], we finally get

$$
\sum_{s_{r-1}=1}^{n} \sum_{s_{r-2}=1}^{s_{r-1}} \cdots \sum_{s=1}^{s_{1}}\left(\begin{array}{c}
\left(\begin{array}{c}
s \\
i+1 \\
m
\end{array}\right) \\
m !
\end{array}\right)=\frac{1}{j=0} \sum_{s=0}^{n} s_{1}(m, j)\left(\begin{array}{c}
r+n-i-s-2 \\
r-1
\end{array}\right)\left(\begin{array}{c}
i+s+1 \\
s
\end{array}\right)^{j} .
$$

The theorem is proved.

\section{Proof of Theorem 3}

We have

$$
\begin{gathered}
S=\sum_{i=1}^{n} \sum_{j=1}^{n}(-1)^{i+j} r_{i j}\left(\begin{array}{c}
q-p-1 \\
q-i-1
\end{array}\right)\left(\begin{array}{c}
m-k-1 \\
m-j-1
\end{array}\right)= \\
=\sum_{i=p}^{q-1} \sum_{j=k}^{m-1}(-1)^{i+j} r_{i j}\left(\begin{array}{c}
q-p-1 \\
q-i-1
\end{array}\right)\left(\begin{array}{c}
m-k-1 \\
m-j-1
\end{array}\right)=\sum_{i=p}^{q-1} \sum_{j=k}^{m-1}(-1)^{i+j} r_{i j}\left(\begin{array}{c}
q-p-1 \\
i-p
\end{array}\right)\left(\begin{array}{c}
m-k-1 \\
j-k
\end{array}\right) .
\end{gathered}
$$

If we replace $i-p, j-k$ by $s, t$, respectively, and put $Q=q-p-1, M=m-k-1$, then we get

$$
S=\sum_{s=0}^{Q} \sum_{t=0}^{M}(-1)^{t+k+s+p} r_{s+p, t+k}\left(\begin{array}{c}
Q \\
s
\end{array}\right)\left(\begin{array}{c}
M \\
t
\end{array}\right)=\sum_{s=0}^{Q} \sum_{t=0}^{M}(-1)^{t+k+s+p} r_{s+p, t+k}\left(\begin{array}{c}
Q \\
s
\end{array}\right)\left(\begin{array}{c}
M \\
M-t
\end{array}\right) .
$$

Let us rewrite the sum $S$ using the formula $\left(\begin{array}{l}n \\ k\end{array}\right)=\operatorname{res}_{x}(1+x)^{n} x^{-k-1}$ and adding terms, which are equal to zero for $s>Q$ and $t>M$ :

$$
S=\sum_{s=0}^{\infty} \sum_{t=0}^{\infty}(-1)^{t+k+s+p} r_{s+p, t+k} \cdot \underset{w}{\operatorname{res}}(1+w)^{Q} w^{-s-1} \cdot \underset{z}{\operatorname{res}}(1+z)^{M} z^{-(M-t)-1} .
$$


The elements $r_{i j}$ are not equal to zero only in the following cases: $i=j, i=j+1, j=i+1$, when $r_{i i}=2, r_{i, i+1}=r_{i+1, i}=-1$. Thus, $r_{s+p, t+k}=2$, if $s+p=t+k$, i.e. $t=s+p-k$ for any $s \geqslant 0$. Note that if $p-k<0$, then the index $t<0$ for $i=0,1, \ldots, p-k-1$. However, in this case, both the binomial coefficient $\left(\begin{array}{c}M \\ M-t\end{array}\right)$ and its integral representation are equal to zero. Continuing this line of reasoning for the cases $r_{i, i+1}=r_{i+1, i}=-1$, we obtain

$$
\begin{aligned}
& S=\sum_{s=0}^{\infty}(-1)^{(s+p-k)+k+s+p} 2 \cdot \underset{w}{\operatorname{res}}(1+w)^{Q} w^{-s-1} \cdot \underset{z}{\operatorname{res}}(1+z)^{M} z^{-(M-(s+p-k))-1}+ \\
& +\sum_{s=0}^{\infty}(-1)^{(s+p-k+1)+k+s+p}(-1) \cdot \operatorname{res}_{w}(1+w)^{Q} w^{-s-1} \cdot \underset{z}{\operatorname{res}}(1+z)^{M} z^{-(M-(s+p-k+1))-1}+ \\
& +\sum_{s=0}^{\infty}(-1)^{(s+p-k-1)+k+s+p}(-1) \cdot \operatorname{res}_{w}(1+w)^{Q} w^{-s-1} \cdot \operatorname{res}_{z}(1+z)^{M} z^{-(M-(s+p-k-1))-1}+ \\
& \quad=\operatorname{res}_{z}\left\{(1+z)^{M} z^{-M+(p-k-1)-1}\left(1+2 z+z^{2}\right) \cdot\left[\sum_{s=0}^{\infty} z^{s} \operatorname{res}_{w}(1+w)^{Q} w^{-s-1}\right]\right\} .
\end{aligned}
$$

Summing over $s$, using the substitution rule and the change $w=z$, we finally get

$$
S=\underset{z}{\operatorname{ras}}\left((1+z)^{M+Q+2} z^{-M+(p-k-1)-1}=\left(\begin{array}{c}
Q+M+2 \\
M-p+k+1
\end{array}\right)=\left(\begin{array}{c}
q-p+m-k \\
m-p
\end{array}\right) .\right.
$$

The theorem is proved.

This work is supported by the Krasnoyarsk Mathematical Center and financed by the Ministry of Science and Higher Education of the Russian Federation in the framework of the establishment and development of regional Centers for Mathematics Research and Education (Agreement No. 075-02-2020-1534/1).

\section{References}

[1] P.Hall, A contribution to the theory of groups of prime-power order, Proc. London Math. Soc., 36(1934), no. 2, 29-95.

[2] M.Hall, Jr., The Theory of Groups, Macmillian, New York, 1959.

[3] E.F.Krause, On the collection process, Proc. Amer. Math. Soc., 15(1964), 497-504.

[4] A.I.Skopin, The Jacobi identity and P.Hall's collection formula for transmetabelian groups of two types, Zap. Nauchn. Sem. Leningrad. Otdel. Mat. Inst. Steklov. (LOMI), 175(1989), 106-112 (in Russian).

[5] V.M.Leontiev, Combinatorial problems connected with P.Hall's collection process, Siberian Electronic Mathematical Reports, 17(2020), 873-889 (in Russian).

[6] S.Kolesnikov, V.Leontiev, G.Egorychev, Two collection formulas, Journal of Group Theory, 23(2020) no. 4, 607-628. DOI: 10.1515/jgth-2019-0074

[7] G.P.Egorychev, Integral Representation and the Computation of Combinatorial Sums, Nauka, Novosibirsk, 1977 (in Russian). Translation of Mathematical Monographs, American Mathematical Society, 1984; 2nd ed. in 1989. 
[8] M.R.Riedel, Egorychev method and the evaluation of binomial coefficient sums, Pnp, mathematic, uni-stuttgard, 2020.

[9] G.P.Egorychev, Yu.M.Gorchakov, The ranks of the factors in the lower central series of a free polynilpotent group, Dokl. Akad. Nauk SSSR, 204(1972), 12-14.

[10] V.M.Leontiev, On Divisibility of Some Sums of Binomial Coefficients Arising From Collection Formulas, Journal of Siberian Federal University. Mathematics \& Physics, 11(2018), no. 5, 603-614. DOI: 10.17516/1997-1397-2018-11-5-603-614

\title{
Интегральное представление и вычисление кратных комбинаторных сумм из холловской теории коммутаторов
}

Георгий П. Егорычев

Сергей Г. Колесников

Владимир М. Леонтьев

Сибирский федеральный университет Красноярск, Российская Федерация

\begin{abstract}
Аннотация. В работе доказывается ряд комбинаторных тождеств, возникших при вычислении показателей степеней коммутаторов в собирательной формуле Ф. Холла. Также вычислена в замкнутом виде сумма, возникшая в результате использования собирательной формулы в группах Шевалле при решении вопроса Б.А.Ф. Верфрица о регулярности их силовских подгрупп.
\end{abstract}

Ключевые слова: интегральное представление, метод коэффициентов, собирательная формула Ф. Холла. 